\title{
RFDM- An Efficient Method for Video Tracking
}

\author{
Karanam Sunil Kumar, N P Kavya
}

\begin{abstract}
Video tracking is a computer vision imperative task. Region-based, feature-based, deformable and model-based (RFDM) tracking algorithms are the four categories of Match-based video tracking type. This survey provides various techniques or methods to object detection, identification and recognition, more light on region-based tracking, dealing with occlusions and overlaps and insufficiencies in model-based tracking and contour model. Finally, various future studies have been recommended at the end of this paper.
\end{abstract}

Keywords: object tracking, Active Contour Models, Content Extraction and Filtering.

\section{INTRODUCTION}

The exponential development in smart working of digital equipment's and online social networking has culminated in large quantities of visual data being collected, in particular the huge and growing accumulation of videos on the Web and social networks. Match based video object tracking is categorized into four types namely region, feature, deformable and model based.

Although many researchers have proposed their research in this area and developed several applications, but still the video tracking remains as challenging are due to variety of applications, different scenarios and involved complexity in tracking of objects in video sequences.

In the proposed literature attention is given to categories of match-based video tracking.

\section{REGION BASED TRACKING}

The area can be perceived in relative motion as the outline of an object's projection in the scene. segmentation of each image is carried out using a movement-based segmentation process and its correspondence was in use between the projected regions and the observations made by the segmentation process. The region can be defined as the outline of the projected object in the frame in relative motion with respect to the camera movement.

Thanks to a numerical regularization strategy, the movement-based segmentation process provides balanced movement-based partitions. This method does not involve direct 3D calculations or the calculation of the area of optic flow unless it is expected.

The portrayal of a zone is not intended to capture the exact boundary. It should provide a depiction of the form and place even in the existence of partial occlusions that helps the tracking task [1]. Using histogram is a common way to capture a less quantity of spatial information among pixels the

Revised Manuscript Received on December 22, 2019.

* Correspondence Author

Karanam Sunil Kumar, CSE, RNS Institute of Technology, Bengaluru, India. Email: sunilkaranam72@gmail.com

Dr N P Kavya, CSE, RNS Institute of Technology, Bengaluru, India. Email: npkavya@gmail.com contribution in formation of histogram bins and generalization of histograms to improve tracking outcomes [2].

The use the original object area of the image as an object model, equate the object model to the contestant image, and are evaluated on the basis of the maximum fitting degree of the math point level is proposed in work related to region grounded tracking [3].

The early region-based tracking algorithms, the object templates are fixed. [4] Introduces the best matching area approach by using the gray image spatial gradient to find the best matching image. In the older region-based tracking techniques, the object templates are fixed. Lucas etal. [4] presents a method to find best matching region by taking grey image spatial gradient to find the image which matches finest. Each and every point is used in the object region's gray gradient values to change the candidate object region. candidate object region uses gray gradient values for each point in the object in the region.

A robust, the adaptive look design of motion-based monitoring adapts to a slowly changing look and retains a realistic indicator of the integrity of the object form while tracking. Through defining stable appearance properties, giving greater weight to estimate movement, less stable properties can be weighted down proportionately [5]. A stable, flexible look design for motion-based monitoring adapts to a slowly changing presence and retains a realistic indicator of the object structure's stability while tracking. Through defining secure appearance properties, giving greater weight to estimate movement, less stable properties can be weighted down proportionately [5]. theoretical model for adaptive appearance.

Zoidi et al., [6] detects using backward subtraction method and objects and Update the object model by similarity to the color histogram. The outcome of the experiment indicates that the tacking proposed succeeds in tracking objects under variations in partial occlusion and scale and rotation.

A standard example of region based tracking algorithms is mean shift algorithm. Mean algorithm of shift [ 7]. The key idea is to build the object model manually or to use the aim detection algorithm. The similarities among object model and object candidate model is described in cost function. The key idea is to use a manual or goal detection algorithm to construct the object model. Setting up a cost function in the goal detection algorithm to elucidate the relation The optimization algorithm is used between the object candidate model and object model to determine the base cost feature and the best cost value in video sequences that match the position of the object.

Because it is easy to implement, it is a classic object tracking algorithm and has a good real-time performance paper $[8,9]$ applies the Mean Shift algorithm to the computer vision field, oodles of improved algorithms $[10,11]$ are proposed which are based on Mean Shift. 


\section{RFDM- An Efficient Method for Video Tracking}

Because of this, global object data used in region-based tracking algorithms uses gray image texture features; they are highly credible. Minor object deformation does not affect the performance of the monitoring, but they are time-consuming as tracking happens in large regions where objects have significant deformations or extreme occlusions leading to a decrease in accuracy, resulting in artifact loss. In recent years, regional monitoring algorithms have focused on how to handle model variations, variations are induced by different poses of moving objects,

The papers $[12,13]$ discuss this problem and have good effects on tracking. When different poses are correctly predicted then only steady monitoring can be done and also this paper discuss this problem and have strong tracking results.

An approach is proposed in paper to remove and use semantic layers from aerial videos to understand scene and track objects [14]. This research also proposes to capture input video from platforms of low flying areas, usually consisting of heavy parallax from non-ground plane structures.

Study also proposes to capture input video from aerial platforms of object flying at a lower attitude, typically consisting of robust parallax from plane structures of non-grounded object. A key aspect of this approach is video frame geo-registration to reference image databases to set up a pixel-in-video geo-spatial coordinate system.
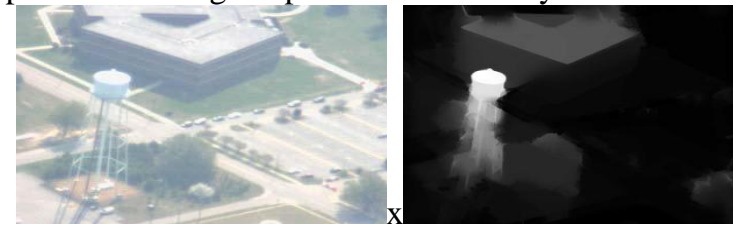

Fig 1a. Input frame

Fig 1b. Depth Cue

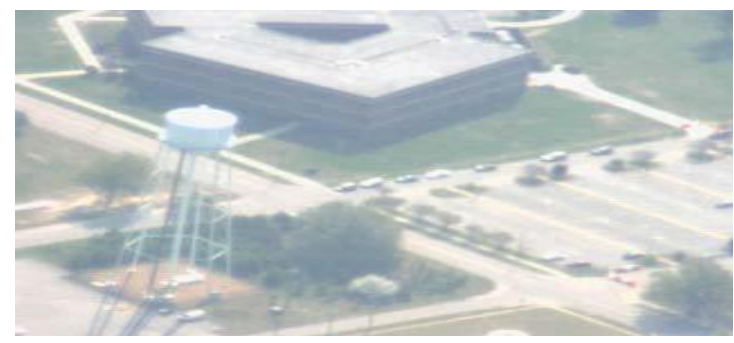

Fig 1c. Aerial video scene segmentation.

After combining video, geo-reference image and geographic information system. Fig 1.a Represents a input frame. Fig 1.b Displays the corresponding video depth cue estimated Fig 1.c Pictures of desired results of scene segmentation.

The cost function process for region-based tracking is achieved using temporarily consistent local color histograms. Using techniques of first-order gradient descent, usually cost functions are generally optimized based on region. A structured derivation of a Gauss-Newton optimization method resulting in significantly faster convergence and extremely accurate and stable tracking performance [15].

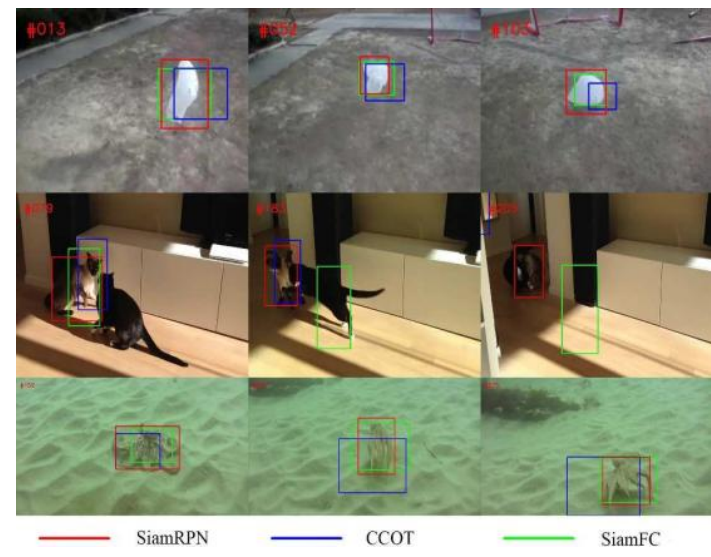

Fig 2: Comparisons of Siam and CCOT and Siam FC approaches.

The Siamese area proposal platform is an end-to-end trained process with images of large scale. It contains of Siamese network for processing of features and proposed region, the subnetwork involves the branch of categorization and the branch of regression. The anticipated model will be developed like a l one-shot detection function in the inference process. The proposed model will be developed as a local one-shot detection function during the inference process. This work pre-calculates the Siamese subnetwork model branch and creates the association layers to perform online tracking as trivial convolution levels. Benefit from the proposal optimization is the standard multi-scale check and it is possible to ignores the digital fine tuning.

A prominent feature of the region-based tracking technique is that by using a predictive color system, the actual target region is discriminated against from the background. From the derived pertinent region, shape model derives that can be merged with color structure that allows the tracker to be stable when the target object's color distribution is identical to other objects. The frame one is time-consuming for online applications, numerous CNN-based tracking methods proposed to segment a target object with in the video sequences. The first frame, consumes lof time for online applications, various CNN-based methods are built to segment a pointed object via video by extensively fine tuning on the image mask. In the present paper [16], A quick and efficient video object segmentation algorithm has been proposed that the segmentation process can begin immediately when the images are received.

\section{FEATURE BASED TRACKING}

The key idea of feature related tracking techniques on image [17] uses the applications to classify the different object and image features of objects in the video sequences. Usually, algorithm consists of two main steps, that include extraction of features and matching features. In the next frame, the most similar object is found out in the second step according to the matching yardstick of the feature. The method can easily determine the object's position with the two steps in video sequences.

By applying certain limitations when moving the feature points, early work solves the issue of identifying the correlating functionality points between both the neighboring frames. E. Sethi et al. 
[18] consider the motion functions on points to be smooth and suggest a theory of related iterative technique and coherence direction.

Rangarajan et al., [19] present an optical flow-based non-iterative polynomial time approximation algorithm to solve the correspondence problems. But these algorithms in the occlusions scene have a high computational complexity and a higher error rate.

Tissainayagam et al., [20] implies a juncture-based object tracking algorithm. The technique looks for the local corner points and defines them as the important points to be tracked by MHT. Nonetheless, it produces proper results for simple structures in geometry due to the difficulty involved in corner point extraction. It has poor dynamic object efficiency.

Li et al., [21] has lately been discovering a new sub-set evaluation method for selecting object tracking features. The review provides a new understanding of how to draw the innovative features and Improve the performance of the real-time algorithm.

Tracking algorithms based on features do not use global object technology. Although the objects have minor occlusions, other visible features can be used to perform the task of tracking. The algorithms are not able to deal successfully with complete occlusions and overlaps. In the area of algorithms related feature based tracking, a numerous article has appeared in recent years. see some of the important papers [22,23]

Recently, favorable performance has been shown by structured output support vector machine (SVM) based tracking algorithms. Even so, their real-time applications are limited by the time-consumption involved in candidate sampling and multifarious optimization. A innovative large margin object tracking method has been proposed in this paper, which shows the strong discriminative capacity of organized output. First, SVM significantly increases the speed of the algorithm for the correlation filter. Furthermore, a multi-model target detection technique is proposed to increase the accuracy of the target location and avoid model drift from similar objects or background noise being implemented. Third, we take advantage of the feedback from results of high confidence tracking to avoid the problem of model corruption. The proposed tracker can be incorporated in two versions: first, using traditional hand-crafted and secondly, using neural networks of convolution (CNN) derived characteristics to verify the algorithm's high functionality.

A novel algorithm for fast and robust stereo optical odometry based on feature selection and tracking (SOFT) achieves drift reduction, is focused on carefully choosing a subset of stable features and tracking them through the pictures. There are different measurements of rotation and translation for two consecutive poses. The five-point method is used to estimate the rotation, while the three-point method is used to estimate the translation [24].

A new, probability-modelled soft data association was used in this study. In an entangled EM model, the association chances are determined with the optical stream equation, which maximizes expectations (marginalization) across all associations. In addition, affine deformations in relation to the initial point were determined to require longer tracks and the resulting residual was used as a measure of durability. The calculated optical flow allows different temporal integration for each feature and size inversely proportional to the flow length [25].

A tracking method based on the combination of point cloud and visual feature is proposed to improve the applicability and robustness of the three-dimensional tracking method of an augmented reality-assisted assembly guidance system for mechanical products. First, a reference point cloud is used to define the tracking benchmark coordinate system to determine the position of the guiding information for virtual assembly. A camera tracking algorithm is then implemented that combines visual matching and point cloud alignment. In order to obtain appropriate matching points of visual features in a texture less assembly sense, a novel ORB function matching technique is implemented based on the accuracy of path vectors. [ 26]

One of the major threats to the structural integrity of steel bridges is fatigue cracks formed under repeated loads. Human testing is the most commonly used method for detecting fatigue break, but it is time consuming, intensive Laborie, and lacks accuracy. In this research, a computer-based approach to fatigue crack detection is suggested using a short video stream taken by a digital camera that is consumer-grade. To detect the surface movement of the tracked structure under repetitive pressure, a tracking technology is applied to the image. A crack detection and localization algorithms are then effectively designed to look for differential features in various video frames induced by the observing of cracks. wo laboratory experiments of fatigue cracks in-plane and out-of-plane, respectively, confirm and test the utility of the suggested method. Results indicate that the proposed method will describe the fatigue crack robustly, even if the crack is surrounded by other crack-like objects, distorted by complicated surface textures, or invisible to human eyes due to crack closure

All patch-based and correlation filter-based tracking approaches have produced competitive results on precision and robustness, but there is still ample room for improving overall performance if the challenging factors in visual tracking are carefully addressed. This paper presents a patch-based tracker that uses multiple effective features to adapt the kernel correlation filters. it takes advantage of the useful data from various parts of the target, each template patch is trained by a kernel correlation filtering method and the weight of each patch in a particle filtering framework is adjusted. Experiments show that this scheme can handle the problem of occlusion effectively [29].

Visual tracking with multiple features was proven to be a comprehensive method. Video sequence having of variations like illumination, occlusion, and pose can occur. One of the key issues in this approach is, how to properly select and combine appropriate image features. New joint sparse representation model for robust fusion proposed in this paper By using the advantages of sparse representation, the proposed technique automatically removes non reliable features to be combined for tracking. To capture the non-linear similarity of features, we extend the proposed method to a general kernelled framework that can fuse features across different kernel spaces. he results is robust tracking performance. 


\section{RFDM- An Efficient Method for Video Tracking}

The qualitative experimental results on publicly available videos show that both sparse representation-based and fusion-based trackers are outperformed by the proposed method [30].

In the area remote sensing, tracking of video sequence is the emerging filed, that refers to online tracking of interested moving objects. In a satellite video image, interested target are only few pixels, even when the train is long. Thus, compared to traditional visual tracking, proposed tracking st faces new challenges like detecting low-resolution targets, not properly presented features, targets with literarily similar background. There has been little research on satellite video target tracking, and to know whether or not existing tracking algorithms can still work on video satellite data [[31].

Because classic cameras sample the film at constant periods, in constant time between subsequent frames will not permit the tracker to fetch more information. For many high-speed robotic and sight applications, it is decisive to provide high-frequency measurement updates. A modern vision system named DAVIS, integrating a conventional camera with an event-based asynchronous detector in the same pixel array, will do this. The DAVIS encodes the video content among two successive frames through an asynchronous stream of events that transmits changes in pixel brightness at microsecond pixel density. his paper presents the first algorithm for detecting and tracking visual features using both the frames and the DAVIS event data. Features are first detected in the gray scale frames and then tracked asynchronously through the stream of events in the constant time intervals.

Spatial-temporal view of the tracked features of DAVIS (frames and events) is shown in below image. Due to their high efficiency and strong robustness, tracking methods based on correlation filter attracted a lot of attention However, a single-function tracker is obviously not enough to adapt to the target's complex appearance changes. Furthermore, in the field of visual tracking, rapid and exact estimation of the scale remains a challenging problem. In this work, we impose an independent scale filter to estimate an object's scale and combine two additional features to further boost the tracker's performance. At the same time, a strategy to reduce the computational load is adopted.

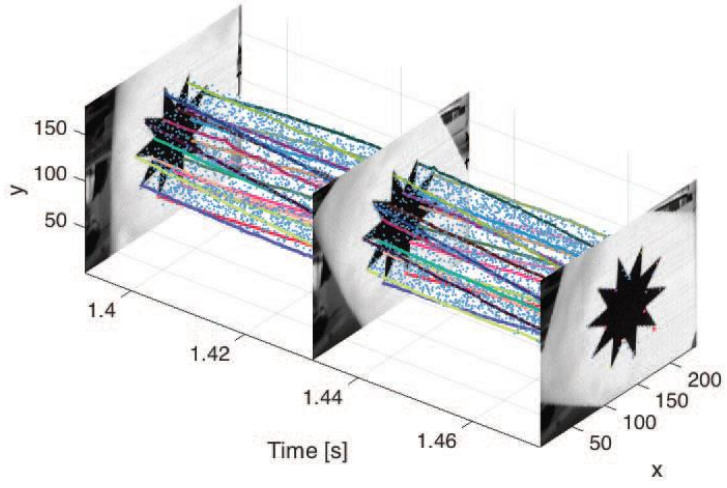

Fig 3: Spatial-temporal view of the DAVIS output.

Finally, a dynamic model update mechanism based on learning rate is inserted to effectively alleviate the problem of model degradation by suppressing the influence of noisy changes in appearance. [32]

\section{DEFORAMABLE BASED TRACKING}

The simple technique of tacking based on deformable templates is proposed in algorithms [33], is to use the exterior or curve with Clear contour elasticity, flexibility and deformation characteristics of the traveling object's contour or edge as a contour-bound object and update the contour to suit the entity.

Snakes are Active Contour models that lock and exactly identify neighboring edges. They describe a Snake as a function that minimizes energy, influenced by outside forces of compulsion and influenced by image forces. There were hints for numerous improved algorithms based on Snake. [ 34,35] I-Snake.

B-spline snakes are tools used to trace the contour easily and intuitively. Start with a theoretical argument for splines which are part of conventional formulation by showing the optimal curvature-constrained snake is a cubic spline, regardless of the shape of the field of external energy. Unfortunately, due to a large number of control points, these regularized snakes suffer from sluggish convergence rate, difficulties in evaluating the factors of weight correlated with the curve's inner energies. So proposed paper suggests an alternate solution where intrinsic size of the spline system is derivable, this leads to a reduction in the parameters to be configured and reduces the requirement of energies for inner B-Snake [36].

To show the ability of the proposed system, the role of controlled texture segmentation is considered. The specified filtering process on textured objects using the filters like isotropic, anisotropic and evaluating their results as multi-component conditional probability density functions produces the textured feature space. Demonstrating the potential of the proposed framework is the task of segmentation of supervised texture is considered. The textured feature space is produced by the process of filtration on the textured objects utilizing isotropic and anisotropic filters and by evaluating their results as contingent probability density functions on multi-component. Texture segmentation is obtained as an enhanced Geodesic Active Contour System, G-Snake, by unifying area and boundary-based information [37].

The main feature of the AI-snake is that during the image extraction process the model contour pose is dynamically changed to match the real snake contour by solving the correspondence question of the snake design and testing the correct affine transformation, AI-Snake [38].

Part-based trackers are useful for the rigorous monitoring of the target object's local data. Unlike other part-based approaches that separate all types of target items into a collection of rectangular fixed patches A novel model has been suggested in this paper in which a series of deformable patches interact dynamically on monitoring non-rigid artifacts. In particular, research proposes a shape-preserved kernel based connection filter (SP-KCF) that can be used for robust tracking purpose-shaped data. The SP-KCF for adaptive analysis of individual patches is integrated into the level-set system. The proposed deformable patches are thus target-dependent, capable of assuming complex topology, and deformable to conform to aim variations [39]. 
In epithelial cell sheets, a novel method for tracking cells connected through a visible network of membrane junctions is common and resembles planar graphs where each face corresponds to a cell. This structure is leveraged by work and a method is developed to track the whole tissue as a deformable graph. This coupled model in which vertices inform the optimal positioning of edges and vice versa captures global tissue component relationships and results in accurate and robust cell tracking [40].

In the cases of sound intrusion, occlusions, and edge distortion, the exact contours of the designs mentioned above are quite difficult to obtain. In the cases of sound intrusion, occlusions, and edge distortion, the exact contours of the designs mentioned above are quite difficult to obtain. Importing prior color knowledge [41], knowledge of texture [42], and knowledge of shape [43] et al., and using them to limit contours to correct edges of items. ilmaz et al suggested contour monitoring method using the approximation of the $\mathrm{k}$ density of kernel and the Gabor wavelet system to direct the evolution of the contour based on the color and texture properties. Ninget al. [44] presents a innovative framework for combined active contour segmentation (JRACS) and registration provides the strong stability for tracking of non-rigid objects.

Template based deformable algorithms, on the other side, simply describe artifacts, minimize computation and strong robustness in partial occlusions cases. Algorithms are hard to start automatically monitoring because it's sensitivity to initialization activity in tracking. Another disadvantage is that the contour rate of tracking precision is reduced. In recent years, the papers [ 45,46$]$ have suggested the state-of - the-art monitoring algorithms focused on deformable mold.

A comprehensive study has been suggested to condemn content in real time. A classy method was proposed to accomplish video denoising in real time. The intrinsic problem of noise removal in real time can be resolved significantly due to the use of different filtering approaches for background and moving object. A comprehensive study has been suggested to condemn content in real time. A temporary average filter is used to restore the context in such a filtering strategy, and a spatial filter is used to reduce the foreground to eliminate noise [47].

\section{MODEL BASED TRACKING}

The basic idea of tracking algorithms related to model based [48] is: defining an object's mathematical model based on prior information, tracking objects by matching the template and object model of the candidate area, then in the monitoring process, the object properties can be calculated by the parameters of the size, the position parameters and the movement parameters. The models can be divided into the following models: hierarchical [49], 2D [50] and 3D [51].

The widely used monitoring technologies are 3D designs. The 3D model's mechanism works by finding the 3D model structure, object model based on previous knowledge by assembling the sequences of video to determine 3D parameters model to obtain the movement's object parameters. Nevertheless, the design of 3D models requires a large number of variables, the template fitting methods are quite complicated, and the time consumption is more in $3 \mathrm{D}$ tracking and contains low real-time performance.
Yang et al., [52] propose a $\mathrm{n}$ model-based vehicle position algorithm for 3D, allowing optimized cameras to accurately and robustly evaluate vehicle poses in traffic scenes the degree is measured by using image end point method to which a pose evaluation function matches the edge points with the projected model.

Yang et al. [54] develop an interactive random conditional field template and the method to convert the tracking process in to an minimization of energy challenge. This method is powerful in distinguishing objects with similar appearances from spatially close. Observation perspectives do not easily affect model-based tracking algorithms, so to different motions they are intrinsically robust. The algorithms are very precise because $3 \mathrm{D}$ data is used. The papers $[55,56]$ focused on how real-time performance has been improved over the past few years.

Detection, tracking and recognition of video-based traffic signs are key components for smart transport systems. Thorough research has shown the various art techniques, particularly techniques of deep learning, may achieve pretty good output on public data sets. Deep learning approaches, however, require extensive resources in computation.

Apart from previous research, this work introduces a unified incremental computational framework to detect, track and recognize traffic signs using a mono-camera placed on a moving vehicle in non-stationary environments [57].

Quick Moving targets are always moved or smeared in various image sequences outside the scene to establish footage via Synthetic Aperture Radar (SAR) ring. In this article, focused on the convolution Neural Network, an innovative moving target monitoring method with tracking (SDT) and shadow detection is proposed. CNN monitoring identification is used on possible moving candidates derived from a series of spatial and temporal sub-opening SAR objects to identify and monitor moving targets based on the shadow feature of moving target in SAR imagery.

Shadow extraction of the moving target is done on SAR first frame, which works like a circular SAR. The circular SAR is shown below in fig.

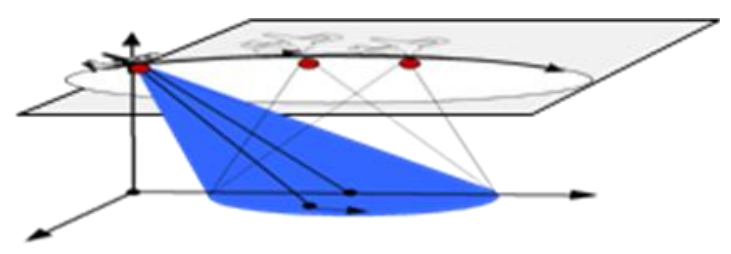

Fig 4. Circular SAR over a 3D video frame

The static targets show different event analysis, while traveling targets represent the different location and concentration. The storage of video on circular SAR information will boost shift detection on object sequence to continue tracking a target or monitoring an interesting area [58]. 


\section{RFDM- An Efficient Method for Video Tracking}

\section{RESULTS AND DISCUSSIONS}

There are numerous challenges in images and videos to detect and recognize objects. This paper focus some prominent challenges specifically related to video in this section. They also discuss potential avenues of video text retrieval technology in particular for prospective literature study. There are numerous challenges in images and videos to detect and recognize objects.

- The researchers must deal with the problems of collecting visual content and realizing that several methods have been suggested in identifying, recording, describing images and recognizing human behaviors. Decrease in computational cost and time reduction.

- Balance between tracking robustness and tracking accuracy.

- Handling of occlusions and overlaps in feature-based tracking.

- Limitations in contour model.

- Betterment in automatic tracking of objects.

- Decrease in computational cost and time reduction.

\section{CONCLUSIONS}

Several algorithms on video object are now being proposed but all most are attributed to application limitations only suitable for particular objects or set scene. Thanks to the nature of tracking objects, the difficulty of situations and the scope of implementations, the current video object tracking algorithms face immense challenges. The solution to existing monitoring technologies is to construct real time visual tracking model having robustness feature. It is difficult to tackle the question of innovation in tracking, in that knowing the expansion of technology by citizens. We also know that there is no standard definition for citizens ' understanding, and it is difficult to establish their mathematical model. Nonetheless, visual object tracking devices have a wide range of use. It has become increasingly convenient to implement whether in theory or in application with the advancement of machine and computational methods.

\section{REFERENCES}

1. Saravanakumar, S.; Vadivel, A.; Saneem Ahmed,C.G., "Multiple human object tracking using background subtraction and shadow removal techniques," Signal and Image Processing (ICSIP), 2010 International conference.

2. Stanley T. Birchfield Sriram Rangarajan Electrical and Computer Engineering Department Clemson University, Clemson, SC 29634.

3. G. D.HagerandP. N.Belhumeur, "Efficient region tracking with parametric models of geometry and illumination", Pattern Analysis and Machine Intelligence, IEEE Transactions, vol.20, (1998), pp.1025-1039.

4. B. D.LucasandT. Kanade,ed.,"An iterative image registration technique with an application to stereo vision",IJCAI,vol.81, (1981), pp.674-679.

5. D.Jepson,D. J.Fleetand T. F.El-Maraghi,"Robust online appearance models for visual tracking",Pattern Analysis and Machine Intelligence, IEEE Transactions, vol.25, (2003), pp.1296-1311.

6. O.Zoidi,A.Tefasand I.Pitas, "Visual object tracking based on local steering kernels and color histograms", Circuits and Systems for Video Technology, IEEE Transactions, vol.23, (2013), pp.870-882.

7. D.ComaniciuandP.Meer,"Mean shift: A robust approach toward feature space analysis",Pattern Analysis and Machine Intelligence, IEEE Transactions, vol.24, (2002), pp.603-619.

8. D.Comaniciu,V.Rameshand P. Meer,eds.,"Real-time tracking of non-rigid objects using mean shift.Computer Vision and Pattern Recognition”, Proceedings. IEEE Conference, (2000).
9. D.Comaniciu,V.Rameshand P. Meer,"Kernel-based object tracking",Pattern Analysis and Machine Intelligence, IEEE Transactions, vol.25, (2003), pp.564-577.

10. 10 . O. D.Nouar, G. Aliand C. Raphael,eds., "Improved object tracking with CamShift algorithm", Acoustics, Speech and Signa Processing, ICASSP Proceedings, IEEE International Conference, (2006).

11. Leichter,"Mean shift trackers with cross-bin metrics",Pattern Analysis and Machine Intelligence, IEEE Transactions, vol.34, (2012), pp.695-706.

12. K. J. Cannonsand R.P. Wildes,"The applicability of spatiotemporal oriented energy features to region tracking",Pattern Analysis and Machine Intelligence, IEEE Transactions, vol.36, (2014), pp.784-796.

13. R.He,B.Yangand N.Sang,"Integral region-based covariance tracking with occlusion detection",Multimedia Tools and Applications, (2014),pp.1_22

14. Jiangjian Xiao Hui Cheng Feng Han Harpreet Sawhney Sarnoff Corporation (jxiao, hcheng, fhan, hsawhney)@sarnoff.com

15. Bo Li1,2, Junjie Yan3, Wei Wu1, Zheng Zhu1 "High Performance Visual Tracking with Siamese Region Proposal Network SenseTime Group Limited

16. Jingchun Cheng, Yi-Hsuan Tsai, Wei-Chih Hung, Shengjin Wang, Ming-Hsuan Yang "The IEEE Conference on Computer Vision and Pattern Recognition (CVPR)”, 2018, pp. 7415-7424

17. S. N.Sinha,J. M.Frahm,M.Pollefeysand Y. Genc, "Feature tracking and matching in video using programmable graphics hardware", Machine Vision and Applications, vol.22, (2011), pp.207-217.

18. K.Sethiand R.Jain,"Finding trajectories of feature points in a monocular image sequence",Pattern Analysis and Machine Intelligence, IEEE Transactions, vol.1, (1987),pp.56-73.

19. K.RangarajanandShah, "Establishing motioncorrespondence",CVGIP image understanding, vol.54, (1991), pp.56-73.

20. P.Tissainayagamand D.Suter,"Object tracking in image sequences using point features",Pattern Recognition, vol.38, (2005), pp.105-113.

21. G.R.Li,Q.M. Huang,J.B.Pang,S.Q. Jiangand L. Qin,“Online selection of the best feature subset for object tracking", Journalof Visual Communication and Image Representation, vol.23, (2012), pp.254-263.

22. E. J.Ongand R.Bowden,"Robust facial feature tracking using shape-constrained multiresolution-selected linear predictors",Pattern Analysis and Machine Intelligence, IEEE Transactions, vol.33, (2011), pp.1844-1859.

23. X. Yangand K. T. Cheng, "Local Difference Binary for Ultrafast and Distinctive Feature Description",Pattern Analysis and Machine Intelligence, IEEE Transactions, vol.36, (2014), pp.188-194

24. .Mengmeng Wang, Yong Liu, Zeyi Huang; "The IEEE Conference on Computer Vision and Pattern Recognition (CVPR)", 2017, pp. 4021-4029

25. Ivan Petrovi' c Department of Control and Computer Engineering Faculty of Electrical Engineering and Computing University of Zagreb Unska 3, 10000 Zagreb, Croatia.

26. Alex Zihao Zhu, Nikolay Atanasov, and Kostas Daniilidis 2017 IEEE International Conference on Robotics and Automation (ICRA) Singapore, May 29 - June 3, 2017

27. Xiangyuan Lan, Shengping Zhang, Member, IEEE, Pong C. Yuen, Senior Member, IEEE, and Rama Chellappa, Fellow, IEEE TRANSACTIONS ON IMAGE PROCESSING, VOL. 27, NO. 4 , APRIL 2018

28. Vision based Fatigue crack detection of steel structures using video feature tracking, computer-aided civil and infrastructure Engineering 33(2018)783-799

29. Robust visual tracking via patch based kernel correlation filters with adaptive multiple feature ensembleWei Chen, Kaihua Zhang, Qingshan Liu , NEUCOM17300, S0925-2312(16)30688-9

30. Joint Sparse Representation and Robust Feature-Level Fusion for Multi-Cue Visual Tracking Xiangyuan Lan, Student Member, IEEE, Andy J. Ma, Pong C. Yuen, Senior Member, IEEE, and Rama Chellappa, Fellow, IEEE

31. Tracking Objects From Satellite Videos: A Velocity Feature Based Correlation FilterJia Shao, Bo Du, Chen Wu, Lefei Zhang, IEEE Transactions on Geoscience and Remote Sensing 12 June 2019. 
32. Xianyou Zeng1,2, Long Xu3, Yigang Cen1,2, Ruizhen Zhao1,2, Shaohai Hu1,2, Guohui Xiao4 This work was partially supported by the National Natural Science Foundation of China (NSFC) under Grants 61872034, 61572067, 61572461, 11790305, 11433006, 61572063,61841503 and 61741507.

33. Y.Zhong,A. K.Jainand M. P.Dubuisson-Jolly, "Object tracking using deformable templates",Pattern Analysis and Machine Intelligence, IEEE Transactions, vol.22, (2000), pp.544-549.

34. Kass et al.,[25] propose Active Contour Models which are the most commonly used deformable templates in object tracking.

35. M.Kass,A.Witkinand D.Terzopoulos,"Snakes: Active contour models",International Journal of Computer Vision, vol.1, (1988), pp.321-331

36. P.Brigger,J.Hoegand M. Unser,"B-spline snakes: a flexible tool for parametric contourdetection",Image Processing, IEEE Transactions, vol.9, (2000), pp.1484-1496

37. N.Paragiosand R.Deriche,"Geodesic active regions and level set methods for supervised texture segmentation", International Journal of Computer Vision, vol.46, (2002), pp.223-247.

38. Z.Xue,S. Z.Liand E. K.Teoh,"AI-EigenSnake: an affine-invariant deformable contour model for object matching”,Image and Vision Computing, vol.20, (2002), pp.77-84

39. Xin Sun, Ngai-Man Cheung, Hongxun Yao, Yiluan Guo; The IEEE International Conference on Computer Vision (ICCV), 2017, pp. 5495-5503]

40. Roger S. Zou and Carlo TomasiIEEE TRANSACTIONS ON MEDICAL IMAGING Volume: 35 , Issue: 7 , July 2016]

41. A.Yilmaz,X.Liand M. Shah, eds.,"Object contour tracking using level sets",Asian Conference on Computer Vision,(2004).

42. N.Houhou, J.Thiranand X.Bresson, eds.,"Fast texture segmentation model basedon the shape operator and active contour",Computer Vision and Pattern Recognition, CVPR,IEEE Conference, (2008).

43. G.Charpiat,O.Faugerasand R.Keriven, eds.,"Shape statistics for image segmentation with prior", Computer Vision and Pattern Recognition,CVPR,IEEE Conference, (2007).

44. J. F.Ning,L.Zhang,D.Zhangand W.Yu, “Joint Registration and Active Contour Segmentation for Object Tracking”,Circuits and Systems for Video Technology, IEEE Transactions, vol.23, (2013), pp.1589-1597.

45. A.Nakhmaniand A.Tannenbaum, "Self-Crossing Detection and Location for Parametric Active Contours", Image Processing, IEEE Transactions, vol.21, (2012), pp.3150-3156.

46. W. M. Hu, X. Zhou, W. Li, W. H. Luo, X. Q. Zhangand S. Maybank, "Active Contour-Based Visual Tracking by Integrating Colors, Shapes, and Motions", Image Processing, IEEE Transactions, vol.22, (2013), pp.1778-1792.

47. Reeja, S. R., \& Kavya, N. P. (2012). Real time video denoising. 2012 IEEE International Conference on Engineering Education: Innovative Practices and Future Trends (AICERA). doi:10.1109/aicera.2012.6306745

48. H. Dahlkamp, H. H. Nagel, A. Ottlikand P. Reuter, "A framework for model-based tracking experiments in image sequences", International Journal of Computer Vision, vol.73, (2007), pp.139-157.

49. A.Karaulova,P. M.Halland A. D.Marshall,"Tracking people in three dimensions using a hierarchical model of dynamics",Image and Vision Computing, vol.20, (2002), pp.691-700.

50. S. Wu, L. Hongand J. R. Layne, "2D rigid-body target modelling for tracking and identification with GMTI/HRR measurements", Control Theory and Applications, IEE Proceedings, vol.151, (2004), pp.429-438.

51. D. M.Gavrilaand L. S.Davis, eds.,"3-D model-based tracking of humans in action: A multi-view approach",Computer Vision and Pattern Recognition, Proceedings CVPR, IEEE Computer Society Conference, (1996).

52. H. Yang, J. Lou, H. Z. Sun, W. M. Huand T. N. Tan,eds., "Efficient and robust vehicle localization ",Image Processing, Proceedings International Conference, (2001)

53. D. T. Chenand J. Yang, "Robust Object Tracking via Online Dynamic Spatial Bias Appearance Models", Pattern Analysis and Machine Intelligence, IEEE Transactions,vol.29, (2007), pp.2157-2169.

54. B.Yangand R.Nevatia,"Multi-Target Tracking by Online Learning a CRF Model of Appearance and Motion Patterns",International Journal of Computer Vision, vol.107, (2014), pp.203-217.

55. X. Li, A. Dick, C. H. Shen, A. van den Hengeland H. Z. Wang, "Incremental Learning of 3D-DCT CompactRepresentations for Robust Visual Tracking", Pattern Analysis and Machine Intelligence, IEEE Transactions,vol.35, (2013), pp.863-881.
56. Wojek, S. Walk, S. Roth, K. Schindler and B. Schiele, -Monocular Visual Scene Understanding: Understanding Multi-Object Traffic Scenes", Pattern Analysis and Machine Intelligence, IEEE Transactions, vol.35, (2013),pp.882-897.

57. An Incremental Framework for Video-Based Traffic Sign Detection, Tracking, and Recognition Yuan Yuan, Senior Member, IEEE, Zhitong Xiong, and Qi Wang, Senior Member, IEEE IEEE TRANSACTIONS ON INTELLIGENT TRANSPORTATION SYSTEMS, VOL. 18, NO. 7, JULY 2017

58. Donghun Yeo, Jeany Son, Bohyung Han, Joon Hee Han; The IEEE Conference on Computer Vision and Pattern Recognition (CVPR), 2017, pp. 1812-1821

\section{AUTHORS PROFILE}

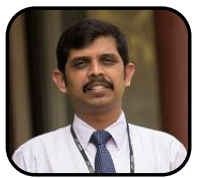

Karanam Sunil Kumar, holds the Bachelor of Engineering in Computer science and Engg. Along with M. Tech degree from VTU Belagavi.

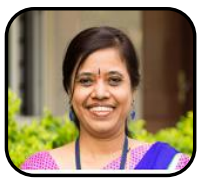

Dr. N P Kavya, holds Bachelor of Engineering in Computer Science and Engg. along with MS in software systems and $\mathrm{Ph}$. D in computer science form VTU Belagavi. She has a vast experience of 24 years in the field education and research. She is currently a Professor in Computer science and Engg., Department, RNSIT, Bengaluru. She has published around 90 research papers in reputed international journals including IEEE, Elsevier, Springier (SCI and Web of Science). Has 94+ citations in Google scholar as on Oct 2019. Her main areas of expertise are Machine Learning, Artificial Intelligence, Big Data analytics 